\title{
BMJ Open Quality Improving diagnostic specimen management systems in an oral medicine department
}

\section{Olivia Barratt (D) , ${ }^{1}$ Melanie Simms (D) , ${ }^{1}$ Miriam John, ${ }^{2}$ Michael Lewis (D) , Phil Atkin (10) ${ }^{1}$}

To cite: Barratt 0, Simms M, John $\mathrm{M}$, et al. Improving diagnostic specimen management systems in an oral medicine department. BMJ Open Quality 2020;9:e000926. doi:10.1136/ bmjoq-2020-000926

Received 16 January 2020 Revised 21 May 2020 Accepted 11 June 2020
Check for updates

(C) Author(s) (or their employer(s)) 2020. Re-use permitted under CC BY-NC. No commercial re-use. See rights and permissions. Published by BMJ.

${ }^{1}$ Oral Medicine, Dental Hospital, Cardiff and Vale University Health Board, Cardiff, UK ${ }^{2}$ Quality Improvement Skills Section, Health Education and Improvement Wales (HEIW), Cardiff, UK

Correspondence to Dr Olivia Barratt; olivia.barratt123@gmail.com

\section{ABSTRACT}

Histological, haematological and microbiological investigations are essential in the field of oral medicine and are a crucial adjunct to clinical findings, often being relied on to obtain a definitive diagnosis. Importantly, in some cases, these investigations can help exclude or confirm the presence of malignancy. This project highlighted some problems regarding labelling and recording of specimens in an oral medicine department and a lack of clear specimen management processes. It aimed to improve specimen management by reducing reported incidents surrounding diagnostic tests. Quality improvement methods such as process mapping were key to understanding the journey of specimens and the departments involved at each stage of the system. Initiatives included a recording log book, staff training, information signage around the clinic and delegation of responsibilities, all of which were implemented over multiple plan, do, study, act (PDSA) cycles. The project was extremely successful and since implementation there has been a clear and sustained reduction in reported incidents. The small number of incidents which did occur all involved transportation of specimens and none involved labelling or recording. One can conclude that the change in test management systems in terms of recording and labelling of specimens in the department has been sustained. Ongoing engagement with stakeholders and senior leaders is the priority to ensure further reduction in incidents in the future and that the improvements are maintained. This project demonstrates how simple, realistic, cost-effective, quality improvement initiatives can have a significant positive impact on patient care and hospital management systems.

\section{PROBLEM}

Histological and microbiological analysis is routinely used in oral medicine to aid clinical diagnosis. Tissue biopsies are employed commonly to identify malignancy or dysplasia. They also aid diagnosis of common oral conditions such as lichen planus and lichenoid reactions, and are essential for diagnosis of bullous diseases including pemphigus and mucous membrane pemphigoid. In the oral medicine department, tissue biopsies are regularly carried out under local anaesthetic. As well as these, microbiological testing is often employed using swabs, imprints and oral rinses for analysis to detect oral pathogens such as Candida albicans and Staphylococcus aureus. Moreover, the department also uses haematological testing to detect conditions which may manifest in the oral cavity such as iron-deficiency anaemia and HIV.

The key role of histological, microbiological and haematological tests in oral medicine diagnosis should not be underestimated. These investigations often exclude, or lead to a positive malignant diagnosis, and can be potentially life saving. The organisational systems in place for the specimens to be taken, transported and delivered to the appropriate lab for testing are crucial for accurate and timely diagnosis. This process involves numerous hospital teams from clinicians, to hospital porters and laboratory staff (figure 1). Any problems in this chain could result in a specimen failing to be analysed. A repeat specimen is often required, meaning duplication of tests for the patient and delays in care which could affect the treatment outcome. There is also the cost implication to consider when repeated tests are required. Patients may have more appointments than were initially needed and this will therefore affect waiting times for others. Mistakes could also lead to an incorrect diagnosis, and a malignant condition failing to be recognised, potentially having fatal consequences for the patient.

Within the oral medicine department, there was anecdotal evidence among team members that there were problems surrounding the specimen samples and their management. An audit found 12 reported incidents on the department from December 2016 to October 2018 (figure 2). It is important to recognise the difference between incidents and issues in this context. The authors took incidents to mean those which were officially reported on the hospital reporting online tool-Datix. The anecdotal evidence in the department of a number of 


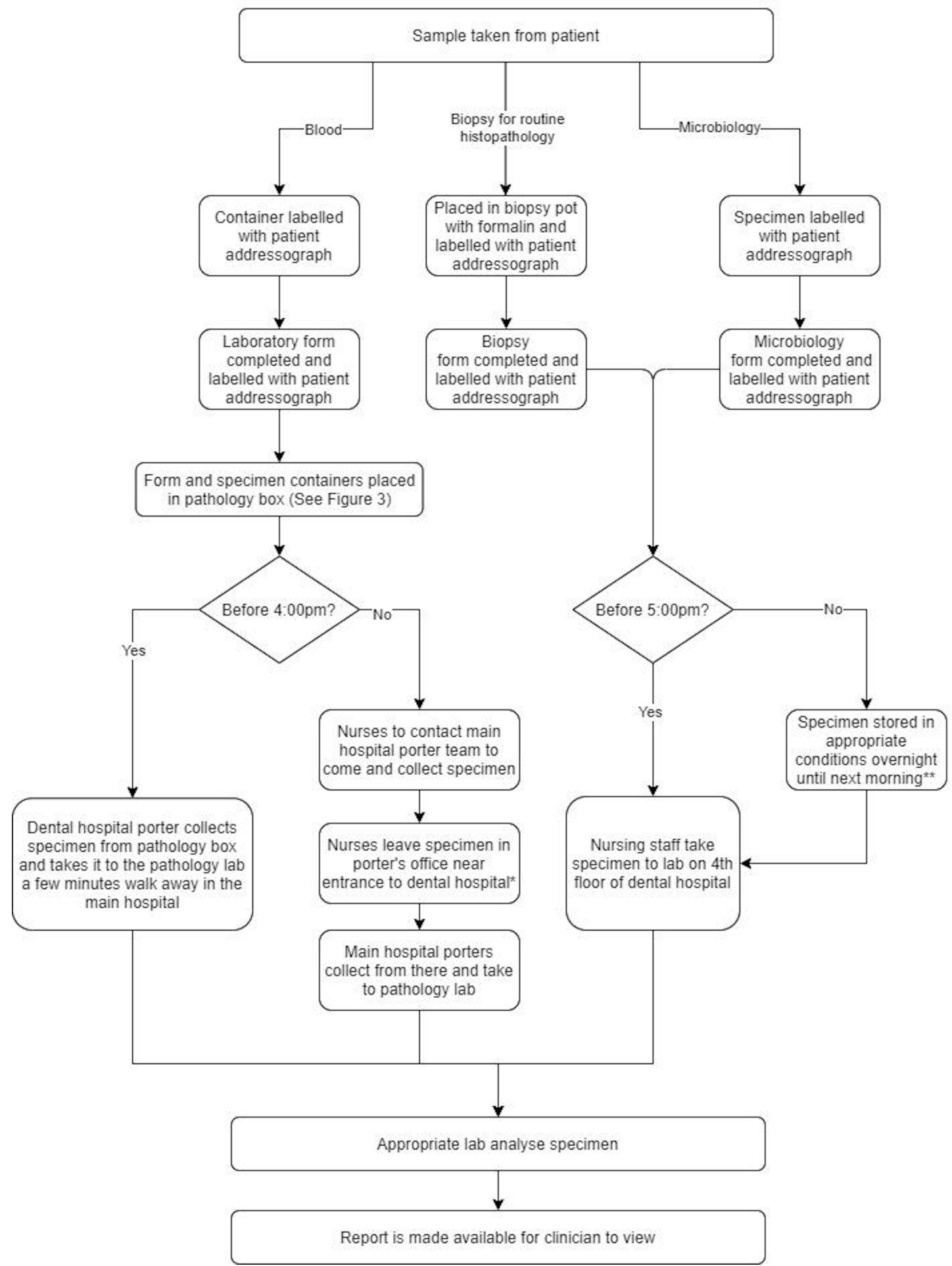

Figure 1 Process map before implementation of new measures. ${ }^{*}$ There were a number of incidents with main hospital porters being unaware of the policy or unavailable for collection due to other duties. ${ }^{*}$ There is no clinic on a Friday afternoon so specimens would always make the Friday 17:00 hours cut-off for the laboratory.

issues with specimen management refers to those which were not reported via official channels and therefore not classed as true incidents. The audit data looked exclusively at official incidents reported on the online system. Six of these incidents involved specimen pots which were not labelled and one incident where a container was labelled incorrectly. These incidents were recognised and rectified immediately and no patients came to any harm. However, these data highlight a key area for improvement to prevent future incidents occurring.

The oral medicine department is based at the University Dental Hospital, Cardiff. The University Dental Hospital consists of a purpose built independent building on the Heath Park site. It is the only unit in Wales that 


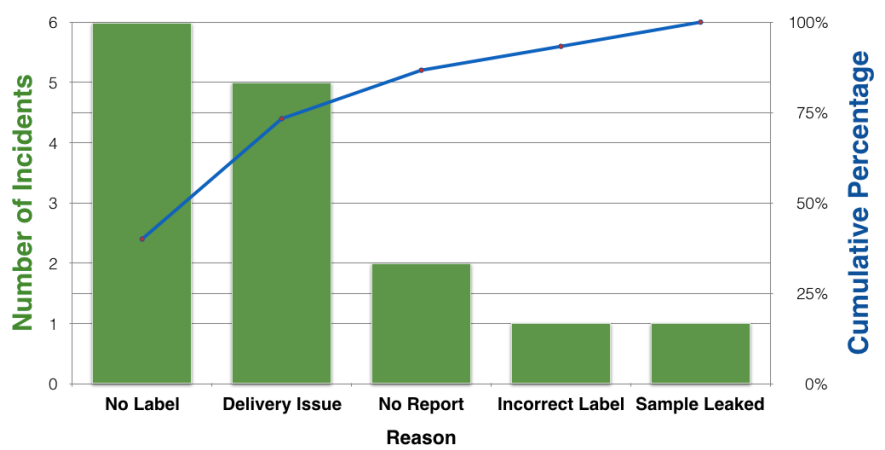

Figure 2 Incidents reported regarding specimens taken on an oral medicine department.

provides services for both the specialist dental treatment of patients and education of dental students. The hospital site treats over 92120 outpatients and 1352 day cases annually. It supports the delivery of education to approximately 220 dental undergraduate students as well as providing support for postgraduate dental education and research. With the Community Dental Service, it forms the Dental Clinical Board of the University Health Board. General practitioners or specialists, both medical and dental, refer patients to the hospital for advice and treatment about oral medicine problems and conditions. Patients can be referred to the department routinely or urgently, for example if malignancy is suspected.

The department is relatively small, led by two consultants, one specialist registrar and a cohort of supporting dental core trainees (DCTs). Most DCTs will rotate every 6-12 months onto other specialties. There is a senior dental nurse responsible for the running of the clinic and normally two dental nurses working on any given oral medicine clinic. There is a core set of oral medicine dental nurses who regularly work on the clinics. However, if these staff members are not working that day due to leave or sickness, then a replacement nurse will be allocated to the clinic from another department. The regular rotation of DCTs and the presence of nurses who do not routinely work on the clinic, was recognised as an issue and potential barrier to change early on in the project.

Each specimen type (blood, microbiology or histology) is sent to the appropriate lab for analysis. Blood is delivered to the pathology laboratory which is a few minutes walk away in the main hospital. This laboratory is open to receive specimens 24 hours a day. The dental hospital porter works between the hours of 9:00 and 16:00 hours and takes the blood specimens during these times. If the specimen needs to be delivered after 16:00 hours, then this is where there can be difficulties. The oral medicine nurses contact the main hospital porter to request that they collect the specimen and deliver it to the lab. Previous audit data showed that this process was not always effective, due to the main hospital porters not being available, having other duties or simply not knowing where the oral medicine department is located.

For microbiology and biopsy specimens, the process is different and somewhat less complex. The lab for these specific tests is in the same building as the oral medicine department, just two floors above. This laboratory is open $9 \mathrm{am}$ until $5 \mathrm{pm}$ and specimens are hand delivered by nursing staff during these hours. If after $5 \mathrm{pm}$, the specimen is stored appropriately overnight and delivered to the lab after 9am the next morning.

\section{BACKGROUND}

Quality improvement initiatives reported in the literature consistently show that electronic methods of recording are key to improving specimen management. ${ }^{1-3}$ There is also valid evidence to show that the use of barcode systems on patient recording labels reduces risk of adverse outcomes. ${ }^{45}$ One study found that most errors in specimen management are made during test request management and labelling, and occur less in laboratory testing facilities. ${ }^{2}$ Others have shown that the use of staff education can be beneficial in improving outcomes regarding specimen management. ${ }^{6}$ The oral medicine department in Cardiff uses physical sticky-back patient labels with barcodes(Addressograph labels).

\section{MEASUREMENT}

The December 2016 to October 2018 audit data showed that there was a clear need for an improvement project which looked at reducing the number of patient incidents and improving patient outcomes. The department staff and laboratory staff were approached and the readiness and enthusiasm for change among regular team members was clear.

For this project, we collected baseline measurements retrospectively using the hospital incident reporting system, Datix. This is an organisation-wide electronic incident reporting system. Initial data collection looked at incidents December 2016 to October 2018. There were 12 incidents reported in this period with one incident on average every 47 days. An ambitious Specific, Manageable, Achievable, Realistic, Time Bound objective was created:

To reduce the number of reported incidents relating to specimen management; they should occur no more than once every 150 days, by the end of October 2019.

At the start of the project, an incident was reported on average every 47 days. Therefore, the new aim sought to set a measurable and realistic target that an incident would occur no more than once every 150 days.

Process mapping allowed the team to understand the journey of the specimen (figure 1). This was then linked back to the audit data to allow a more comprehensive view of the process to appreciate where potential mistakes could occur (figure 2). The following potential sources of incidents were considered:

- Clinicians forgetting to label or mislabelling specimen container. 


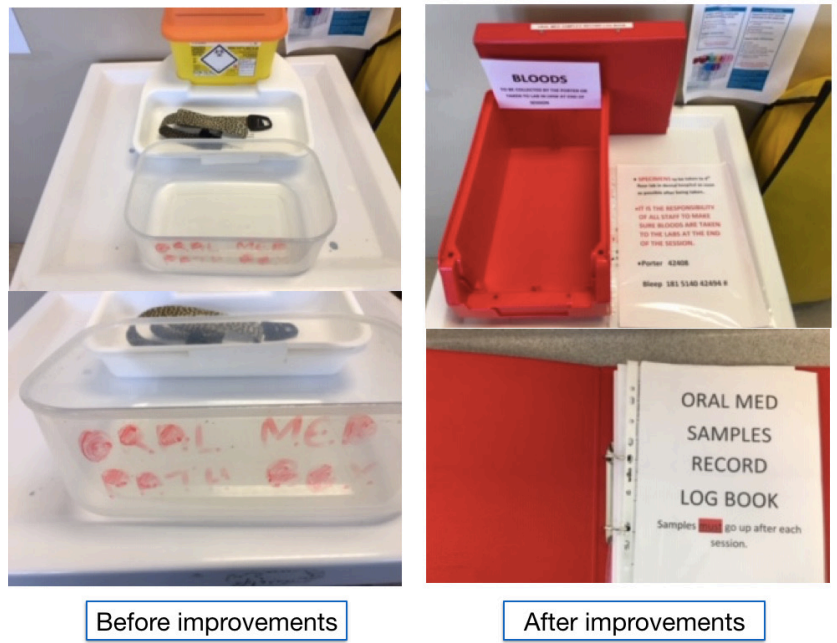

Figure 3 Improved and clearer signage at specimen collection point.

- Laboratory form not being properly completed (missing patient label or missing mandatory written information) or labelled with incorrect patient label.

- One box on clinic for all specimen container types (blood, microbiology and histopathology) meaning often not all blood specimens are collected by the porter (they are hidden beneath other containers and forms, so are missed).

- Dental hospital porter does not arrive to clinic to collect specimens, or staff do not contact him to advise that a collection is required.

- Staff unfamiliar with location of pathology lab and so specimens are not delivered to correct place.

- Main hospital porter does not come to collect specimen (after 16:00 hours or dental hospital porter on annual leave).

- No record of specimen being taken.

Each step of the mapped process was clear. However, the team questioned just how aware every team member was of these stages. For those staff who had recently joined the department, there was a lack of clarity concerning their role and requirements within the process. It was also clear that not all nursing staff were aware of their role to contact the main hospital porter team for blood specimens after 16:00 hours. The information signage around clinic was limited (figure 3 ). For any nursing staff members allocated to the clinic with limited experience in that specific department, they were often unaware of the procedures in place. Interestingly, the vast majority of incidents involved specimens and forms simply not being labelled with patient details.

After considering the reasons for the problems, using tools such as a Barrier-Aids Analysis and the 5 Whys, ideas were discussed and chosen. These changes were implemented independently of each other. One change was made every 3 months using repeated PDSA cycles and measured in terms of the individual success of that change. Measurement was made via the number of reported incidents at the end of each PDSA cycle. Any changes deemed to have limited positive impact or indeed negative impact were reconsidered and improvement cycle continued.

\section{DESIGN}

The project lead initiated the project as part of a Health Education and Improvement Wales (HEIW) supported Silver Quality Improvement training. ${ }^{7}$ This programme, unique to Wales, offers National Health Service staff free, structured, high-quality training in the core principles of quality improvement. Resources and trainees are available to offer support for projects carried out as part of the course. Potential ideas for change were discussed with the department staff and also with HEIW Quality Improvement teaching staff. An ease/benefit matrix was developed to assess the potential of each idea and the team's perceived sphere of influence was also recognised in the decision-making process:

\section{Nurse training and awareness}

Change would involve education sessions regarding the new process during clinic time. Potentially positive benefits and longevity were clear as the nursing team are permanent members of staff, in contrast to DCTs who rotate between departments usually every 6 months.

\section{Electronic test request system}

There is clear evidence to show the beneficial use of electronic systems to improve specimen management. ${ }^{1-3}$ The authors agreed this measure was beyond the scope of implementation due to financial constraints. For this reason, this measure was not considered, as more costeffective and realistic options were available to measure first. It is important to note that in the department, the results of microbiology tests are sent back from the lab via an electronic system and accessed by the clinician electronically. For bloods and histology, a paper report is always delivered to the consultant and must be checked and signed before making its way to the clinic/patient folder, as well as an electronic report being available.

\section{Recording log book}

This initiative would address some of the incidents where specimens have gone missing. With no formal recording system in place, there is no way the department can track any specimens in an attempt to locate them. The idea of a $\log$ book is potentially very easy and cost-effective to implement. Attention must be paid to recording of patient data in a safe and secure way. Challenges may include staff adherence especially on busy clinics.

\section{Improved signage around clinic}

Creating clear signs and labels for storage of samples has potential benefits for clinical staff and also hospital porters who transport specimens to the appropriate laboratory. This initiative is relatively straightforward, inexpensive and has clear longevity requiring little to no maintenance. Challenges were considered including 
changing the signs if the processes change. However, it was agreed this was a potentially useful measure.

\section{Changes to hospital porter staff shift patterns and availability}

There were a significant number of incidents occurring involving specimen transportation and hospital porters being unavailable to collect specimens and deliver to the appropriate labs. A recurrent problem is that the dental hospital porter finishes his shift at 16:00 hours and the oral medicine clinic runs until 17:00 hours, sometimes later. Microbiology and biopsy specimens are able to be stored in an appropriate space and can be delivered the next morning. However, haematological specimens were more problematic as a $12+$ hour delay means that the blood is not able to be analysed accurately. Any blood specimens obtained from patients after 16:00 hours were at risk of missed collection and late delivery to the laboratory, rendering them potentially useless and unable to be accurately analysed. Although all agreed that this change would have huge benefits, the project team felt that extending porter hours was out of their circle of influence. It may be a change to consider in the future and would certainly involve engaging with senior hospital leaders. However, a smaller more feasible change was developed. This involved DCTs delivering the specimens to the appropriate laboratories themselves if needed, after 16:00 hours. Challenges to this change centred around staff having very little knowledge of the exact location of the laboratory on the hospital site and the biannual turnover of DCTs.

\section{Raise porter awareness of the problem and provide further training}

Involving peripheral team members is potentially a very useful strategy. However, without the porters being available to deliver specimens after 16:00 hours, further training would still not change their hours of availability. There is one specific porter who works in the dental section of the hospital. On the days he is not working, the specimens are supposed to be collected by porters who work all over the extensive hospital site. Given the scale of the hospital site and vast porter team, providing training for such a large number of staff whom the project team did not know how to contact, was deemed at this preliminary stage to be difficult to implement. However, it was considered a potentially useful initiative for the future if required after other changes had been implemented and tested.

\section{STRATEGY}

\section{PDSA cycle 1}

Change: Recording log book (figure 4).

Date: 1 November 2018 to 31 January 2019

Measured: 1 incident occurred during cycle.

Implementation of a $\log$ book showed a clear reduction in incidents reported which was encouraging. It enabled the department to be able to trace all specimens obtained

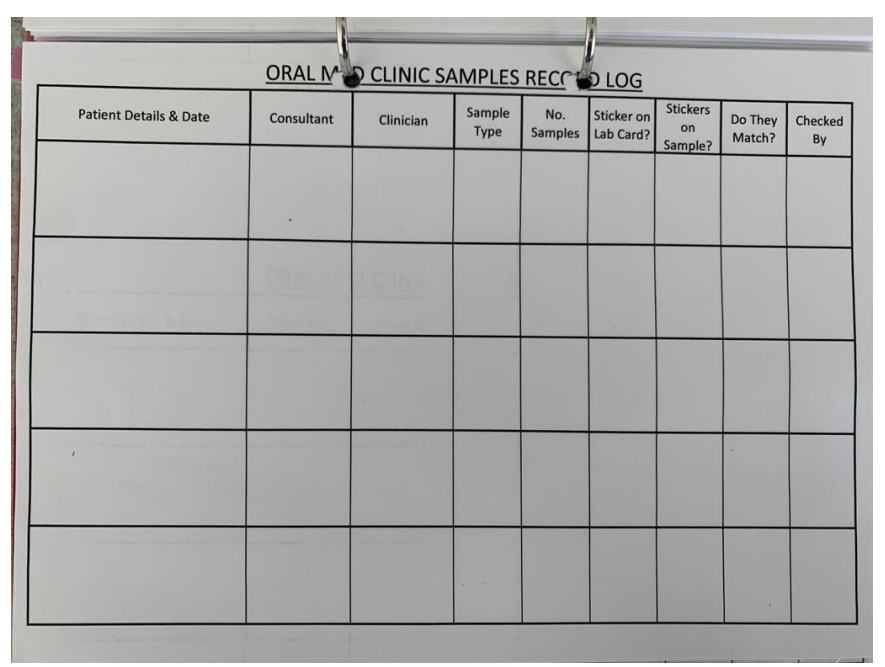

Figure 4 Recording Log Book.

and generally improved awareness of the issue. This initiative was agreed to be continued and implemented into the system of specimen management on the department. Despite a significant positive change, one incident occurred during this cycle and for this reason further PDSA cycles were carried out.

\section{PDSA cycle 2}

Date: 1 February 2019 to 31 April 2019

Change: Informal nurse training sessions

Measured: 1 incident occurred during cycle.

Informal nurse information and training sessions were welcomed by the team and had a positive impact on the staff members. This initiative was deemed useful in improving understanding of the new system on the department and was agreed to be continued. Despite the significant improvements and reduction in problems since the start of the project, this cycle still saw one incident occur. As the aim statement of an incident reported no more than every 150 days was not met, further PDSA cycles were completed.

Notably, this cycle had taken place in March. March and September see the DCT staff members rotate departments in the hospital. The authors suspected this change in staff may be a causative factor in incidents occurring due to lack of awareness and training regarding test management systems. However, with just one reported incident during this cycle, this suggests that the change over in DCTs in March was not necessarily a causative factor in recording errors. Examination of baseline data of previous years during this changeover period showed no clear correlation between DCT start dates and increased incidents. For this reason, it is unclear whether rotation of staff has any clear impact on specimen management.

\section{PDSA cycle 3}

Date: 1 May 2019 to 31 July 2019

Change: Improved and clearer signage at specimen collection point (figure 3)

Measured: No incidents occurred during cycle. 
The addition of information signs around the clinic informing staff of the process showed a clear positive impact on staff and raised awareness of the new systems in place. It is worth noting at this point, that no incidents had occurred relating to specimen labelling and recording, showing the positive impact of the initiatives thus far. This change was simple and cost-effective.

The two incidents which had occurred thus far were both regarding specimen collection by hospital porters. As mentioned earlier, the dental hospital porter is only available until 16:00 hours. The two incidents involved specimens taken after 16:00 hours remaining uncollected. This issue was highlighted and discussed as a barrier to improvement. For this reason, the final PDSA cycle looked at resolving this issue using clinical staff to deliver specimens directly to the lab if taken after 16:00 hours.

\section{PDSA cycle 4}

Date: 1/August 2019 to 31 October 2019

Change: DCTs to deliver specimens taken after 16:00 hours to the appropriate laboratory on-site.

Measured: 2 incidents occurred during this cycle.

This initiative encouraged staff members to deliver specimens to the appropriate laboratories if received after 16:00 hours. This change was relatively difficult to implement as new DCTs as started work on the department every 6 months. Giving certain staff specific duties is difficult to implement if not initiated during formal staff induction.

Notably, both the incidents in this cycle involved transportation of specimens to the appropriate laboratory. In fact, they both occurred in the same week, just 48 hours apart, in the first month that the new DCTs started working in the department. Going forward, it has now been agreed that the nursing staff (who do not change departments regularly) are responsible for taking the specimens two times a day to try and avoid errors made by new DCTs.

\section{RESULTS}

The results were analysed and developed into run charts using the number of days between incidents as a key measure of new initiatives (figure 5). Completed cycles

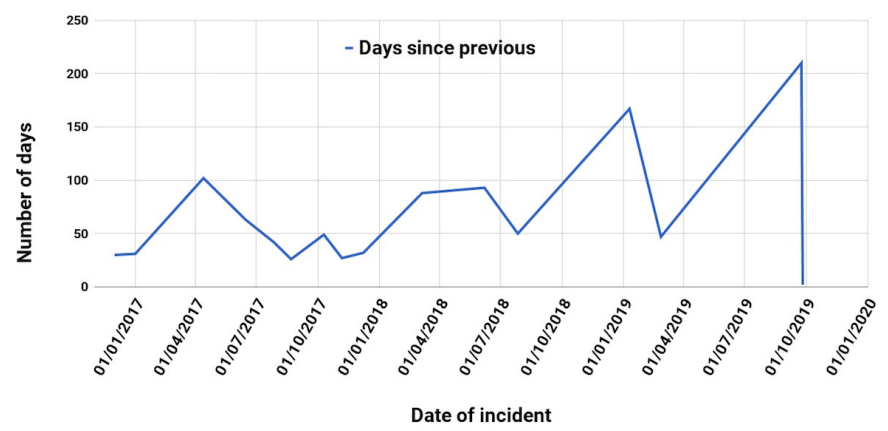

Figure 5 Run chart showing days between reported incidents. showed a clear and sustained improvement in the problem and a clearly positive impact on the system. Implementation of a $\log$ book for recording and tracking specimens, increasing signage and information around the department and informal nurse training have all been effective initiatives. Following interventions, there has been a clear reduction in the number of incidents relating to specimen labelling in the department. Indeed, the run chart demonstrates how after the initiation of the project the average number of days between incidents was above 150 and so the aim statement was met.

Since the start of the project there have only been four incidents in the department and they have all been related to specimens obtained and needing to be delivered to the lab after 16:00 hours when porters are often unavailable. There is still a clear issue in terms of porter collection and the department have worked on a solution to this with key stakeholders. It has now been agreed that after 16:00 hours, the nursing staff will be responsible for taking the specimens to the correct laboratory. This removes the confusion over porter availability and also the point that DCTs who are new to the department may not be aware of the systems in place.

\section{LESSONS AND LIMITATIONS}

This project has highlighted a problem which, if not rectified, could have had major consequences for all stakeholders. A missed malignancy or lost biopsy would cause significant patient distress and also could be potentially catastrophic to patients. There is also the cost benefit of improving specimen management, meaning fewer tests are needed to be repeated. Continued stakeholder engagement was key to the sustainability of the initiatives. Indeed, it was clear that the department was ready for change and this enthusiasm has certainly driven the project to success. However, enthusiasm should not be relied on in the long term. Instead, the changes were embedded into the organisational structure of the department. The new log book and recording system have become engrained into the system of specimen management (figure 6). By implementing new processes, the project has seen significant improvements in patient care and avoided future adverse outcomes. A range of quality improvement tools were used throughout the project. Audit showed the extent of the problem and pareto charting demonstrated potential causes of the incidents. Process mapping was key to understanding the process involved in the specimen analysis and the variety of different teams engaged in the specimen journey. From the process map, a root cause analysis was then carried out to highlight potential reasons for the problem. Stakeholder analysis noted the groups who could contribute to resolving the problem and a driver diagram considered potential changes for improvement. A run chart has usefully enabled data presentation and comparison using the number of days between incidents as a means of analysis (figure 5). 


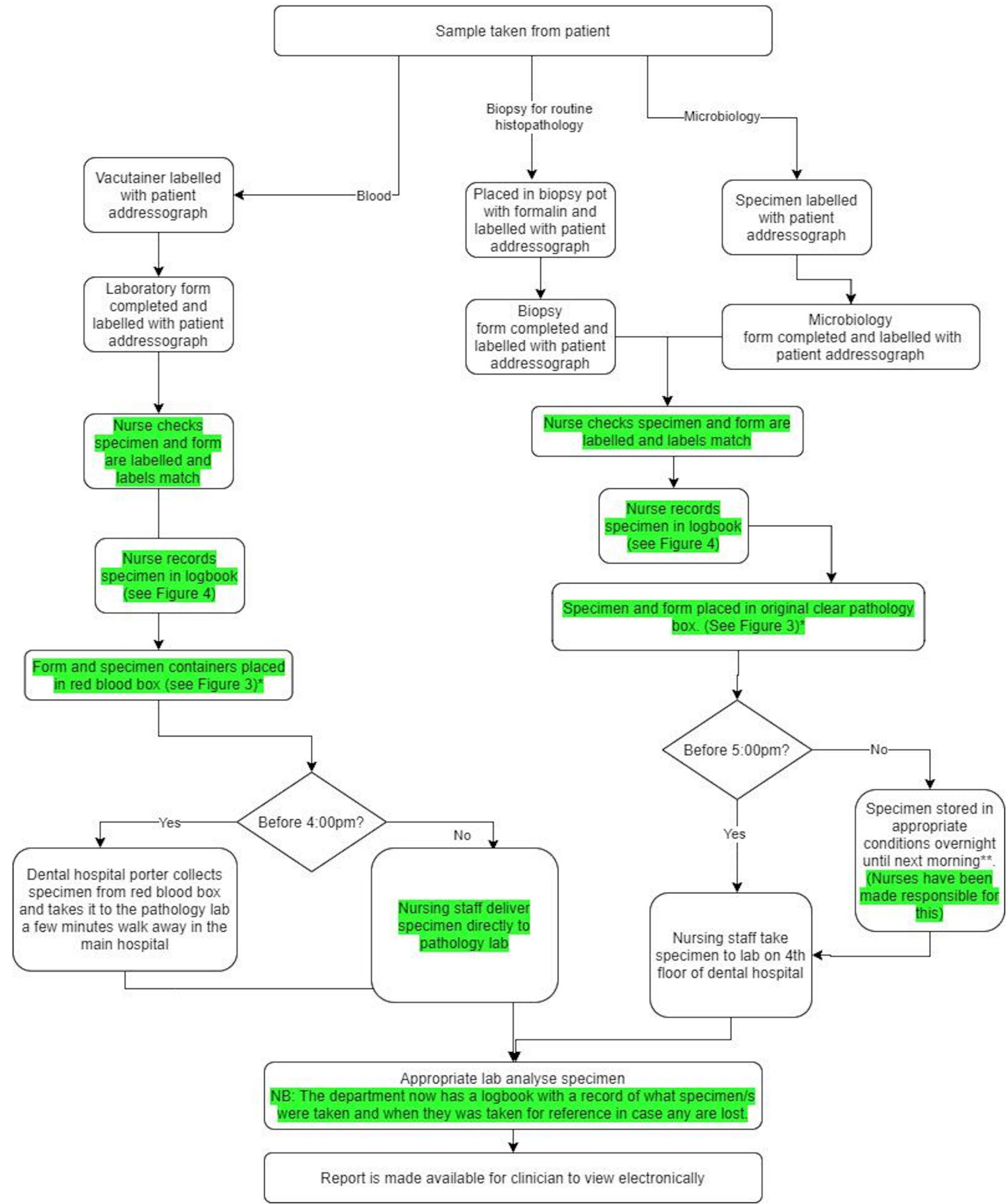

Figure 6 Process map after implementation of new measures. ${ }^{\star}$ The clear box for microbiology and biopsies is now kept apart from the red box for blood specimens. The red box is more visible for porters, and kept in a central location on clinic, to avoid specimens being forgotten and potentially not delivered to the lab in time. ${ }^{* \star}$ There is no clinic on a Friday afternoon so specimens always make the Friday 17:00 hours cut-off for the laboratory.

It is plausible that fewer incidents regarding delivery of specimens would occur if dental hospital porters were available after 16:00 hours or if clinical staff knew where to take these specimens. Extending the dental hospital porters hours would be an effective change. However, a simpler and more cost-effective solution was to ensure clinical staff knew where to take the specimens. Giving the DCTs this role was trialled but did not seem to be effective in the first few weeks of their placement. It is has now been revised and agreed that nursing staff are to deliver these specimens. As no incidents have been reported since this implementation, the ideas has been a clear success.

On reflection, the authors feel it would have been useful to involve peripheral team members and senior organisational leaders from the preliminary phase of the project. Their input may have been beneficial particularly in the process mapping stages and when considering barriers to change. The project recommends that future changes needed should engage peripheral stakeholders such as senior hospital organisational leaders as they would require consultation before any significant 
changes, for example, involving porter hours, would be considered. The project did acknowledge human factors as a potential barrier and the initiatives such as signage around the department addressed this. Future initiatives could involve development of a checklist specific to the department to again address the potential for human error within any system. Widening the project to a hospital wide initiative would be of great benefit to patients and is something the project team would look to for the future to ensure maximum benefit to patient care.

It is important to consider longevity of the improvements in the future. Barriers to this may include new DCTs in the department being unaware of the procedures. Introducing structured staff introductory training for DCTs is an initiative which could address this to ensure departmental awareness of the process and future sustainability.

\section{CONCLUSIONS}

The project was a success and improved specimen management using cost-effective, realistic, team-based measures. It has demonstrated that continuous quality improvement methods and simple initiatives can have a hugely beneficial effect on patient care. The project has now narrowed the focus of any future PDSA cycles and will seek to involve senior organisational leaders to improve patient outcomes further as needed.

Twitter Miriam John @mimbleberry

Acknowledgements Claire Legge, Dental Nurse, Oral Medicine Department, University Dental Hospital, Cardiff. Ewart Johnsone, Quality Improvement Lead, HEIW.Sue Stokes, Quality Improvement Lead, HEIW.

Contributors $\mathrm{OB}$ conceived, designed and led the improvement project and wrote the manuscript. MS and MJ contributed to the writing and editing of the final manuscript. MS, ML and PA implemented the planned actions.

Funding This project was supported by Health Education and Improvement Wales as a Silver Improving Quality Together Project during Dental Core Training.
Competing interests None declared.

Patient and public involvement Patients and/or the public were not involved in the design, or conduct, or reporting, or dissemination plans of this research.

Patient consent for publication Not required.

Provenance and peer review Not commissioned; externally peer reviewed.

Data availability statement All data relevant to the study are included in the article.

Open access This is an open access article distributed in accordance with the Creative Commons Attribution Non Commercial (CC BY-NC 4.0) license, which permits others to distribute, remix, adapt, build upon this work non-commercially, and license their derivative works on different terms, provided the original work is properly cited, appropriate credit is given, any changes made indicated, and the use is non-commercial. See: http://creativecommons.org/licenses/by-nc/4.0/.

ORCID iDs

Olivia Barratt http://orcid.org/0000-0002-9075-5110

Melanie Simms http://orcid.org/0000-0002-3854-5183

Michael Lewis http://orcid.org/0000-0003-1917-0651

Phil Atkin http://orcid.org/0000-0001-6718-8106

\section{REFERENCES}

1 Jakes A, McCue E, Cracknell A. Improving mid stream urine sampling: reducing labelling error and laboratory rejection. BMJ Qual Improv Rep 2014;3. doi:10.1136/bmjquality.u204759.w2219. [Epub ahead of print: 19 Sep 2014].

2 Wallin O, Söderberg J, Van Guelpen B, et al. Preanalytical venous blood sampling practices demand improvement - a survey of testrequest management, test-tube labelling and information search procedures. Clinica Chimica Acta 2008;391:91-7.

3 Granata J. Getting a handle on specimen mislabeling. J Emerg Nurs 2011;37:167-8.

4 Morrison AP, Tanasijevic MJ, Goonan EM, et al. Reduction in specimen labeling errors after implementation of a positive patient identification system in phlebotomy. Am J Clin Pathol 2010;133:870-7.

5 Quillen K, Murphy K. Quality improvement to decrease specimen mislabeling in transfusion medicine. Arch Pathol Lab Med 2006;130:1196-8.

6 Fitzgibbons PL. Challenges in improving Preanalytic specimen handling of routine cancer biospecimens. Arch Pathol Lab Med 2019;143:1300-1.

7 Silver IQT. Wales Deanery, 2020. Available: https://www.walesdeanery. org/quality-improvement-skills-training-qist/silver-iqt [Accessed 9 May 2020]. 